\title{
Stroke Is Different in Systemic Lupus Erythematosus: Implications for Survival and Functionality
}

\author{
Marios Rossides ${ }^{1}$ (iD
}

Undoubtedly, individuals with systemic lupus erythematosus (SLE) are at higher risk for developing cerebrovascular disease than counterparts from the general population without SLE. In a metaanalysis of studies from around the world, the likelihood of individuals with SLE developing both ischemic and hemorrhagic stroke (intracerebral and/or subarachnoid hemorrhage) was more than 2 times that of the general population $^{1}$. Risks for stroke appear to be highest soon after SLE diagnosis, and concomitant antiphospholipid syndrome (APS) in these patients does not markedly exacerbate risks for ischemic stroke ${ }^{2}$.

As stroke commonly occurs at a younger age in SLE than in the general population ${ }^{2,3,4}$, shorter survival and impaired functionality after stroke will impose a greater burden on a patient with SLE than a patient from the general population. Little is known, however, on poststroke outcomes in SLE from prospective studies. One of the few available studies is an investigation from Sweden ${ }^{5}$ in which registers, including a stroke register with universal coverage, were used to identify strokes and their outcomes in individuals with and without SLE. In this study, patients with SLE had a 1.4-fold higher risk for death (from any cause) after the onset of ischemic stroke compared to individuals without SLE 5 . Relative risks for 1-year mortality were even higher for hemorrhagic stroke (2.3-fold higher) $)^{5}$. In addition, SLE was associated with a $73 \%$ higher risk of functional dependence in daily activities 3 months after ischemic stroke onset ${ }^{5}$.

Results from one study may not be generalized to all settings and populations because stroke characteristics and care for these patients may differ among populations. Studies from other SLE populations were therefore warranted. In the article published in this issue of The Journal, Tsoi and colleagues ${ }^{6}$ aimed to examine

${ }^{1} M$. Rossides, $M D, M S c, P h D$ Candidate in Clinical Epidemiology, Clinical Epidemiology Division, Department of Medicine Solna, Karolinska Institutet, Stockholm, Sweden.

$M R$ declares no conflicts of interst.

Address correspondence to Dr. M. Rossides, Karolinska Institutet, Department of Medicine Solna, Clinical Epidemiology Division, Karolinska University

Hospital T2, 17176 Stockholm, Sweden.Email:marios.rossides@ki.se. differences in stroke imaging patterns, poststroke survival, functional impairment, and the occurrence of other adverse outcomes among ethnic Chinese individuals with and without SLE.

They conducted a cohort study consisting of 40 individuals with SLE and 120 age- and sex-matched non-SLE comparators with ischemic (including transient ischemic attack) and hemorrhagic stroke. Patients were identified from a Hong Kong hospital database using the International Classification of Disease codes with enrollment from 1997 to 2017. A medical chart review confirmed diagnoses and allowed for the extraction of clinical information and the assessment of stroke imaging patterns on computed tomography or magnetic resonance scans, as well as stroke severity. Participants were followed for all-cause death and their poststroke functional status was assessed using the modified Rankin Scale ${ }^{7}$. Stroke recurrence and other adverse outcomes related to stroke were also reported. It should be noted that the small sample size did not allow for definite conclusions to be drawn from all analyses performed. Nevertheless, descriptive analyses provided useful insights into features of stroke in this SLE population.

The main findings of this study were as follows:

- Ischemic stroke was more common in SLE than in non-SLE comparators ( $90 \%$ vs $63 \%$, respectively) and stroke in the SLE group was more likely to be severe at presentation.

- Ischemic strokes were more likely to be extensive in SLE than non-SLE, and border zone and multiple infarcts were more commonly observed in the SLE group.

- The 30-day mortality was slightly higher in SLE compared to non-SLE, but differences between the 2 groups were more apparent during the long term (of more than 8 years of follow-up), during which $15 \%$ versus $3 \%$ died in each group, respectively.

- One in 3 individuals with SLE were functionally dependent in their daily activities at 3 months poststroke compared to $8 \%$ in comparators, which translated to 5 -fold increased odds of functional impairment associated with SLE. Neither SLE activity nor APS appeared to be associated with a worse functional outcome.

See Outcome of stroke in SLE, page 533 
- Stroke recurrence and seizures were markedly more frequent in SLE than non-SLE (23\% vs 3\%), whereas neurosurgical interventions were less commonly performed in SLE.

Similar to the previous study from Sweden 5 , 85-90\% of strokes in SLE in the latest study ${ }^{6}$ were of ischemic etiology. However, in the study by Tsoi, $e t a l^{6}$, an overrepresentation of ischemic stroke was observed in the SLE population compared to the general population. During the period Tsoi, et al enrolled patients (1997-2017), hemorrhagic stroke should have accounted for up to $45 \%$ of strokes in this population ${ }^{8,9}$. Because this observation may affect treatment of stroke in SLE, it is worth looking for the reasons for the difference in stroke type between SLE and non-SLE.

It is possible that the discordance observed by Tsoi, et al ${ }^{6}$ is a result of the study design. It may be that cerebral hemorrhage was more likely to result in premature death in patients with preclinical (yet undiagnosed) SLE than in comparators without SLE. In studies restricted to survivors of stroke, which is a prerequisite to investigate stroke outcomes in SLE, we cannot exclude the possibility that ischemic stroke is overrepresented because individuals with SLE who would have developed hemorrhagic stroke died before having the chance to enter the study. Another reason is that in the study by Tsoi, et al ${ }^{6}$, the average patient with stroke irrespective of SLE status was 45 years old. Matching on age may have led to an overrepresentation of hemorrhagic stroke in the non-SLE group because hemorrhage is generally more common than cerebral ischemia at a younger age in this population ${ }^{10}$.

On the other hand, it is likely that ischemic stroke is truly more predominant in SLE than expected. Although more common in SLE, hypertension, which is a powerful triggering factor of hemorrhage, may be better controlled in the SLE group, resulting in fewer hemorrhagic strokes. Other factors such as accelerated atherosclerosis are then allowed to prevail in SLE. Indeed, Tsoi and colleagues ${ }^{6}$ indicate that atherosclerosis was notably more severe in the SLE group, an observation that is in line with several previous studies ${ }^{11}$. Moreover, cerebral vascular disease and secondary APS, which are common features of SLE, are both associated with higher occurrence of cerebral ischemia in this group ${ }^{12,13}$. Nevertheless, further larger studies are needed to disentangle the differences in stroke type in that population.

In the study by Tsoi, et al $^{6}$, not only was ischemic stroke more common than hemorrhagic stroke, it was also more extensive and severe in individuals with SLE than those without. This finding is in contrast to the Swedish study, in which severity of ischemic and hemorrhagic stroke was similar in the SLE and non-SLE groups 5 . A higher proportion of individuals with past strokes in the SLE group (15\% vs 6\%) in the study by Tsoi and colleagues ${ }^{6}$ may have slightly influenced these results, but cannot wholly explain the differences in severity and their association with a higher risk of death and disability in SLE. By meticulously analyzing stroke imaging patterns in their cohort, the authors showed that border zone infarcts and infarcts at multiple locations were observed in more than $60 \%$ of patients with SLE compared to $16 \%$ in the non-SLE group. In the general Asian population, border zone infarcts are found in about $10 \%$ of patients (though their frequency increases with age) and the internal subtype is associated with worse functional outcome at 3 months ${ }^{14}$. It is unknown why border zone and multiple infarcts are so common in SLE. One could hypothesize that distinct atherosclerotic and other cerebrovascular inflammatory phenomena including secondary APS in SLE may play a role ${ }^{12,15,16}$. These findings highlight the fact that we should be vigilant and intervene early to lessen the effects of atherosclerosis and inflammation to prevent unfavorable outcomes in these patients.

Moreover, Tsoi and colleagues ${ }^{6}$ found higher relative risks of functional impairment at 3 months and premature death associated with SLE than those previously observed in the Swedish population ${ }^{5}$. Some of the differences in estimates between studies may have been influenced by the small sample size in the first study and the restriction to stroke survivors in the latter (for the investigation of functional impairment at 3 months). In addition, the higher proportion of individuals with a history of stroke and those with current severe stroke in SLE than non-SLE also contributed to these findings. Taking it a step further, Tsoi and colleagues ${ }^{6}$ could indicate that, contrary to expectation, baseline SLE disease activity and secondary APS could not explain the worse disability status in SLE. It is now well established that most strokes occur during periods of high disease activity and rather close to SLE diagnosis ${ }^{2,17,18}$. Thus, experts advocate primary and secondary prevention measures to be administered early and focus on controlling disease activity (e.g., with corticosteroids, hydroxychloroquine, and/or other immunosuppressants when necessary) and on anticoagulation $^{17,19,20}$. If replicated in larger samples, the findings by Tsoi, et $a l^{6}$ may alter our understanding of the underlying processes in poststroke recovery in SLE and potentially change our approach to primary and secondary prevention of stroke and its consequences in these patients.

In conclusion, stroke remains an overwhelming complication that disproportionately affects survival and daily life activities of individuals with SLE. Although the study by Tsoi and colleagues $^{6}$ furthers our understanding of stroke in SLE, there remains a lot left to learn. Our goal should be to prevent stroke from occurring in patients with SLE and when this is unavoidable, to improve survival and lessen its effect on quality of life. Further epidemiological research is needed to expand the work initiated by Tsoi, et al ${ }^{6}$. We should now aim to identify which interventions (e.g., screening, pharmaceutical, or other) that, if administered in a timely manner, are likely to benefit our multifold scope of prevention. To successfully direct interventions on those who are likely to benefit from them, we need to learn more about how stroke affects diverse SLE populations.

\section{REFERENCES}

1. Holmqvist M, Simard JF, Asplund K, Arkema EV. Stroke in systemic lupus erythematosus: a meta-analysis of population-based cohort studies. RMD Open 2015;1:e000168.

2. Arkema EV, Svenungsson E, Von Euler M, Sjöwall C, Simard JF. Stroke in systemic lupus erythematosus: a Swedish population-based cohort study. Ann Rheum Dis 2017;76:1544-9.

3. Ward MM. Outcomes of hospitalizations for myocardial infarctions and cerebrovascular accidents in patients with systemic lupus erythematosus. Arthritis Rheum 2004;50:3170-6. 
4. Krishnan E. Stroke subtypes among young patients with systemic lupus erythematosus. Am J Med 2005;118:1415.

5. Rossides M, Simard JF, Svenungsson E, Von Euler M, Arkema EV. Mortality and functionality after stroke in patients with systemic lupus erythematosus. J Rheumatol 2017;44:1590-6.

6. Tsoi LK, Mok CC, Man BL, Fu YP. Imaging pattern and outcome of stroke in patients with systemic lupus erythematosus: a case-control study. J Rheumatol 2021;48:533-40.

7. van Swieten JC, Koudstaal PJ, Visser MC, Schouten HJ, van Gijn J. Interobserver agreement for the assessment of handicap in stroke patients. Stroke 1988;19:604-7.

8. Wang Y, Zhou L, Guo J, Wang Y, Yang Y, Peng Q, et al. Secular trends of stroke incidence and mortality in China, 1990 to 2016: The Global Burden of Disease Study 2016. J Stroke Cerebrovasc Dis 2020;29:104959.

9. Zhang LF, Yang J, Hong Z, Yuan GG, Zhou BF, Zhao LC, et al; Collaborative Group of China Multicenter Study of Cardiovascular Epidemiology. Proportion of different subtypes of stroke in China. Stroke 2003;34:2091-6.

10. Ma P, Zhou J, Wang S, Li T, Fan X, Fan J, et al. Differences of hemorrhagic and ischemic strokes in age spectra and responses to climatic thermal conditions. Sci Total Environ 2018;644:1573-9.

11. Schoenfeld SR, Kasturi S, Costenbader KH. The epidemiology of atherosclerotic cardiovascular disease among patients with SLE: a systematic review. Semin Arthritis Rheum 2013;43:77-95.

12. Kaichi Y, Kakeda S, Moriya J, Ohnari N, Saito K, Tanaka Y, et al. Brain MR findings in patients with systemic lupus erythematosus with and without antiphospholipid antibody syndrome. Am J Neuroradiol 2014;35:100-5.

13. Valdés-Ferrer SI, Vega F, Cantú-Brito C, Ceballos-Ceballos J, Estañol B, García-Ramos G, et al. Cerebral Changes in SLE with or without antiphospholipid syndrome. A case-control MRI study. J Neuroimaging 2008;18:62-5.
14. Yong SW, Bang OY, Lee PH, Li WY. Internal and cortical border-zone infarction: clinical and diffusion-weighted imaging features. Stroke 2006;37:841-6.

15. Sibbitt WL Jr, Brooks WM, Kornfeld M, Hart BL, Bankhurst AD, Roldan CA. Magnetic resonance imaging and brain histopathology in neuropsychiatric systemic lupus erythematosus. Semin Arthritis Rheum 2010;40:32-52.

16. Csépány T, Bereczki D, Kollár J, Sikula J, Kiss E, Csiba L. MRI findings in central nervous system systemic lupus erythematosus are associated with immunoserological parameters and hypertension. J Neurol 2003;250:1348-54.

17. Pamfil C, Fanouriakis A, Damian L, Rinzis M, Sidiropoulos P, Tsivgoulis G, et al. EULAR recommendations for neuropsychiatric systemic lupus erythematosus vs usual care: results from two European centres. Rheumatology 2015;54:1270-8.

18. Govoni M, Bombardieri S, Bortoluzzi A, Caniatti L, Casu C, Conti F, et al; Italian Society of Rheumatology. Factors and comorbidities associated with first neuropsychiatric event in systemic lupus erythematosus: does a risk profile exist? A large multicentre retrospective cross-sectional study on 959 Italian patients. Rheumatology 2012;51:157-68.

19. Tektonidou MG, Laskari K, Panagiotakos DB, Moutsopoulos HM. Risk factors for thrombosis and primary thrombosis prevention in patients with systemic lupus erythematosus with or without antiphospholipid antibodies. Arthritis Care Res 2008;61:29-36.

20. Kernan WN, Ovbiagele B, Black HR, Bravata DM, Chimowitz MI, Ezekowitz MD, et al; American Heart Association Stroke Council, Council on Cardiovascular and Stroke Nursing, Council on Clinical Cardiology, and Council on Peripheral Vascular Disease. Guidelines for the prevention of stroke in patients with stroke and transient ischemic attack: a guideline for healthcare professionals from the American Heart Association/American Stroke Association. Stroke 2014;45:2160-236. 
